\title{
Prognostic Significance of IGF-1 Signalling Pathway in Patients With Advanced Non-small Cell Lung Cancer
}

\author{
IOANNIS KOTSANTIS ${ }^{1}$, PANAGIOTA ECONOMOPOULOU ${ }^{1}$, AMANDA PSYRRI ${ }^{1}$, \\ EIRINI MARATOU ${ }^{2}$, DIMITRIOS PECTASIDES ${ }^{3}$, HELEN GOGAS ${ }^{4}$, NIKOLAOS KENTEPOZIDIS ${ }^{5}$, \\ GIANNIS MOUNTZIOS ${ }^{5}$, GEORGE DIMITRIADIS $^{6}$ and STAVROULA GIANNOULI ${ }^{7}$
}

\author{
${ }^{1}$ Section of Medical Oncology, Department of Internal Medicine, \\ Faculty of Medicine, National and Kapodistrian University of Athens, Attikon University Hospital, Athens, Greece; \\ ${ }^{2}$ Hellenic National Center for the Research, Prevention and Treatment of Diabetes Mellitus \\ and its Complications (H.N.D.C), Athens, Greece; \\ ${ }^{3}$ Oncology Section, Second Department of Internal Medicine, Hippokration Hospital, Athens, Greece; \\ ${ }^{4}$ First Department of Internal Medicine, Laiko General Hospital, \\ National and Kapodistrian University of Athens School of Medicine, Athens, Greece; \\ ${ }^{5}$ Department of Medical Oncology, 251 Airforce General Hospital, Athens, Greece; \\ ${ }^{6}$ Second Department of Internal Medicine and Research Institute, Faculty of Medicine, \\ National and Kapodistrian University of Athens, University General Hospital Attikon, Athens, Greece; \\ ${ }^{7}$ Second Department of Internal Medicine, Medical School, \\ National and Kapodistrian University of Athens, Athens, Greece
}

\begin{abstract}
Background/Aim: Insulin-like growth factor 1 (IGF-1)-mediated molecular pathway has been implicated in non-small cell lung cancer (NSCLC) pathogenesis and progression. We aimed to evaluate serum levels of IGF-1, $I G F-2$ and IGF-binding protein 3 (IGF-BP3) before and after standard treatment in patients with advanced NSCLC and their prognostic and predictive correlations. Patients and Methods: Seventy-three patients were prospectively included. Analysis and quantification of circulating levels of IGFI, IGF2, IGFBP3 were performed by total ELISA in peripheral blood samples at baseline and 3 months post-treatment. Results: The median values of IGF-1 and IGF-1/IGF-BP3 ratios (125.82 vs. $133.4 \mathrm{ng} / \mathrm{ml}, p=0.087$ and $0.01006 \mathrm{vs}$. $0.01252, p=0.011)$ were both decreased after treatment. Importantly, the post-treatment value of the ratio was significantly reduced only among responders to treatment (0.01044 from 0.01255, $p=0.02)$. Conclusion: Reduction of IGF-1/IGF-BP3 ratio was statistically significant only among
\end{abstract}

Correspondence to: Prof. Amanda Psyrri, MD, Ph.D., Section of Medical Oncology, Department of Internal Medicine, Faculty of Medicine, National and Kapodistrian University of Athens, Attikon University Hospital, 1st Rimini St, 12462, Haidari, Athens, Greece. Tel: +30 2105831691, e-mail: psyrri237@yahoo.com

Key Words: IGF-1, IGF-2, IGF-BP3, non-small cell lung cancer, chemotherapy. patients with NSCLC who responded to first-line treatment. If validated in larger cohorts, IGF-1/IGFBP3 might be a useful predictive tool for response to chemotherapy in NSCLC.

Lung cancer is the leading cause of cancer-related death worldwide, with non-small cell lung cancer (NSCLC) accounting for approximately $85 \%$ of all cases (1). Despite recent advances in treatment due to the advent of targeted therapies and immunotherapy, the prognosis of patients with NSCLC remains poor (2). The elucidation of molecular pathways involved in the oncogenic process of NSCLC and the discovery of new prognostic and/or predictive biomarkers may improve outcome prediction and thus knowledge-guided treatment.

The insulin-like growth factor-mediated signaling pathway is an important metabolic regulator involved in a number of physiological processes and has also been suggested as an important oncogenic mediator in various neoplasias, including lung, thyroid, laryngeal and esophageal cancer (3). The IGF axis consists of the three ligands insulin, IGF-1, IGF-2, the corresponding cellular receptors IGF-IR and IGF-IIR, and their binding soluble plasma proteins (IGFBPs). These pathways activate PI3K/Akt and Ras/Raf/MAPK kinases, which mediate cell proliferation, survival and metabolism (4). Ligand binding may also regulate various antiapoptotic pathways which inhibit caspase activation. Furthermore, IGFIR may activate antiapoptotic (Bcl-2) and inhibit proapoptotic (Bax, Bcl-xs) members of Bcl-2 family (5). 
Table I. Baseline characteristics of patients.

\begin{tabular}{|c|c|c|c|}
\hline & Responders (\%) & Non-responders $(\%)$ & $p$-Value \\
\hline Patients & 47 & 26 & \\
\hline Age (years) median (quartiles) & $69(62,77)$ & $66(62.75,69.25)$ & 0.13 \\
\hline Male & $39(83)$ & $20(76.9)$ & 0.55 \\
\hline Smoking history & $37(78.7)$ & $23(88.5)$ & 0.36 \\
\hline Ethanol abuse & $5(10.6)$ & $4(15.4)$ & 0.71 \\
\hline Histology & & & 0.6 \\
\hline Adenocarcinoma & $21(44.7)$ & $13(50)$ & \\
\hline Squamous cell carcinoma & $14(29.8)$ & $9(34.6)$ & \\
\hline Other & $12(25.5)$ & $4(15.4)$ & \\
\hline Metastatic at diagnosis & $32(68.1)$ & $17(65.4)$ & 0.81 \\
\hline 1st Line Chemotherapy & & & 0.88 \\
\hline Platinum based non-Bev & 37 (78.7) & $22(84.6)$ & \\
\hline Platinum based with Bev & $6(12.8)$ & $2(2.7)$ & \\
\hline Non-Platinum based non-Bev & $2(4.3)$ & $2(2.7)$ & \\
\hline Non-Platinum based with Bev & $1(2.1)$ & $0(0)$ & \\
\hline Other tyrosine kinase inhibitor & $1(2.1)$ & $0(0)$ & \\
\hline Radiotherapy & $7(14.9)$ & $4(15.4)$ & 1 \\
\hline EGFR & & & 1 \\
\hline Wild type & $12 / 14(85.7)$ & $2 / 14(14.3)$ & \\
\hline Mutant & $6 / 7(85.7)$ & $1 / 7(14.3)$ & \\
\hline OS (months), median (quartiles) & $10(7,14.5)$ & $8(6,11)$ & 0.06 \\
\hline PFS (months), median (quartiles) & $7(5,10)$ & $3(2,5)$ & $<0.001$ \\
\hline
\end{tabular}

Bev: Bevacizumab; EGFR: epidermal growth factor receptor, PFS: progression-free survival; OS: overall survival.

Among IGFs, IGF-1 and its binding protein IGFBP-3 are the most commonly associated with increased lung cancer risk (6). Most of the circulating IGF-1 is bound to the soluble plasma protein IGFBP-3, while a very small percentage of IGF-1 remains in an unbound and biologically active form. Consequently, the bioavailability and bioactivity of IGF1 is controlled by IGFBP3; IGF1 may be "neutralized" after binding with IGFBP3, thereby its mitogenic and antiapoptotic properties are inhibited (7). Therefore, the IGF-1/IGFBP3 ratio is considered an indicator of IGF-1 bioavailability. High serum levels of IGF-1 have been correlated with increased risk for lung cancer, while high levels of IGFBP3 have been associated with decreased risk for the disease $(6,8)$. Moreover, in vitro studies have shown that IGF-1 promotes lung cell growth and invasion, suggesting a role of the IGF-1 pathway in the oncogenic growth of NSCLC (9). On the other hand, interactions between IGF axis and other signaling pathways may lead to resistance to several targeted therapies, such as cisplatin (10) and tyrosine-kinase inhibitors (11). Finally, the IGF-I/IGFBP3 axis has been shown to affect response to treatment and has been implicated in resistance to both chemotherapy and radiotherapy in patients with NSCLC (12).

Although preclinical data suggest that IGF axis is a good therapeutic target for NSCLC, clinical trials using targeted monotherapies against IGF-IR have been disappointing thus far (13). Moreover, the value of IGF-I and IGF-2 as prognostic and predictive biomarkers has not been established yet. Based on these considerations, we aimed to evaluate serum levels of IGF-1, IGF-2 and IGFBP3 before and after treatment in patients with locally advanced/metastatic NSCLC and their potential correlation with clinical outcomes, such as response to therapy and patient survival.

\section{Materials and Methods}

Study design. Seventy-three $(\mathrm{n}=73)$ patients with locally advanced/ metastatic NSCLC were prospectively included in the current analysis. Written informed consent was obtained from all patients before participating in the study. The present study was approved by the Medical Ethical Committee of Attikon University hospital (Athens, Greece) and complies with the principles of the Declaration of Helsinki.

Eligible patients were adults with histologically or cytologically confirmed stage IIIB/IV (14) or medically inoperable NSCLC, ECOG Performance Status (PS) $\leq 2$, life expectancy of more than 3 months and adequate organ function, based on standard laboratory tests including hematology, serum chemistry and coagulation. Major exclusion criteria were prior treatment for NSCLC, type II diabetes mellitus because it could influence IGF levels, and other concomitant neoplasms in the last five years, with the exception of non-melanoma skin carcinomas. Stage was determined according to the TNM classification. Staging examinations included computed tomography (CT) scan of the thorax, abdomen and brain. Bone scan was performed based on symptoms. Patients were enrolled before initiation of treatment.

Patients received chemotherapy, combined chemoradiotherapy (for those with clinical stage of IIIB at diagnosis) or targeted 
Table II. Protein expression values before and after treatment in responders and non-responders

\begin{tabular}{lccc}
\hline Variable Median (quartiles) & Responders, $\mathrm{mg} / \mathrm{ml}$ & Non-responders, $\mathrm{mg} / \mathrm{ml}$ & $p$-Value \\
\hline Pre IGF-1 & $148.35(95.23,191.93)$ & $117.01(76.63,179.36)$ & 0.25 \\
Pre IGF-2 & $619.65(521.25,763.22)$ & $580.48(484.56,734.88)$ & 0.40 \\
Pre IGF1-BP3 & $12077.60(7916.53,16487.48)$ & $10471.45(7658.53,15069.20)$ & 0.40 \\
Pre IGF1/IGF-BP3 & $0.01255(0.00846,0.02004)$ & $0.01225(0.00687,0.01817)$ & 0.58 \\
Post IGF-1 & $130.80(88.98,180.10)$ & $98.00(47.74,175.64)$ & 0.08 \\
Post IGF-2 & $629.41(474.04,785.81)$ & $635.63(278.96,792.93)$ & 0.36 \\
Post IGF1-BP3 & $12077.60(7602.90,19913.10)$ & $13743.10(6461.25,15902.00)$ & 0.54 \\
Post IGF1/IGF-BP3 & $0.01044(0.00629,0.01766)$ & $0.00678(0.00423,0.01364)$ & 0.05 \\
\hline
\end{tabular}

IGF: Insulin growth factor; IGF-BP: insulin growth factor binding protein.

therapy based on disease stage and molecular profile of the tumor. Chemotherapy consisted of a platinum-based or a non-platinumbased regimen with or without bevacizumab, according to the treating physician. Assessment of response to treatment was performed every 3 cycles of treatment. Depending on response, patients were subdivided into responders and non-responders. Responders had either stable disease (SD), partial response (PR) or complete response (CR) based on RECIST 1.0 criteria (15). Patients with stable disease were included in the responders' group because the majority derived clinical benefit from the treatment. Nonresponders were patients who had progressive disease based on RECIST criteria and were switched to second line therapy.

Blood sample collection. Plasma levels of IGF1, IGF2 and IGFBP3 in the peripheral blood were assessed at two separate time points: Blood samples were obtained at baseline and 3 months posttreatment, to allow evaluation of the treatment effect on the levels of these proteins.

Blood Samples were centrifuged at $5000 \mathrm{rpm}$ for $10 \mathrm{~min}$ and plasma was subsequently collected and stored at $-80^{\circ} \mathrm{C}$.

Enzyme-linked Immunosorbent Assay (ELISA) for quantification of protein levels. Quantification of circulating levels of IGF1, IGF2, and IGFBP-3, were performed by total ELISA technique (total enzyme-linked immunosorbent assay). Total IGF ELISA is a quantitative one-step sandwich type immunoassay (Ansh Labs, Webster, TX, USA).

Statistical analysis. Analysis was performed in the total patient population as well as in the subgroups of responders and nonresponders. Univariate analysis was performed between responders and non-responders regarding patient demographics and values of IGF-1, IGF-2, IGF-BP3, and IGF-1/IGFBP-3 ratio, pre- and posttreatment individually, as well as the difference between pre- and post- treatment values for each of the aforementioned treatment groups. The $\chi^{2}$ or the Fisher's exact tests were used, when appropriate, in comparisons of categorical variables, whereas the $t$ test or the Mann-Whitney $U$-test were used in comparisons of continuous variables, depending on the normality of distribution. Wilcoxon test was used for the pre- and post-treatment comparison. Normality was assessed with the Kolmogorov-Smirnov or ShapiroWilk methods, when appropriate. Correlations between variables were assessed with non-parametric Kendall's tau. A $p$-value of
Table III. $p$-Values related to variation in protein expression values before and after treatment.

\begin{tabular}{lccc}
\hline Pre-Post comparison & Total & Responders & Non-responders \\
\hline IGF-1 & 0.08 & 0.25 & 0.18 \\
IGF-2 & 0.82 & 0.75 & 0.99 \\
IGF1-BP3 & 0.86 & 0.42 & 0.69 \\
IGF-1/IGF1-BP3 & 0.01 & 0.02 & 0.31 \\
\hline
\end{tabular}

IGF: Insulin growth factor; IGF-BP: insulin growth factor binding protein.

$\leq 0.01$ was considered as indicative of statistical significance. Comparisons and analyses were performed with the SPSS software (IBM SPSS Statistics for Windows, Version 22.0. Armonk, NY: IBM Corp).

\section{Results}

The study included 73 patients with stage III/IV inoperable NSCLC treated in the Medical Oncology Unit of Attikon University Hospital, Athens, Greece, from January 1st, 2014 through December 31th, 2017. Of those, 59 (80.8\%) were males. Median age was 67 years.

Patient characteristics and treatment. Sixty (82.2\%) out of 83 patients had a history of smoking. Regarding tumor pathology, approximately forty-six percent of patients (34/73) had adenocarcinoma, one third $(23 / 73,31.5 \%)$ had squamous cell carcinoma and the remaining patients had other histology types $(16 / 73,21.9 \%)$. Two-thirds of patients $(67.1 \%)$ had metastatic disease at the time of diagnosis. Response to first-line treatment was documented in 47 patients $(64.4 \%)$. Regarding the type of chemotherapy, most patients were treated with platinum-based regimens without bevacizumab $(59 / 73,80.8 \%)$. The remaining patients received either platinum-based regimens with bevacizumab $(8 / 73,11 \%)$ or non-platinum-based regimens $(5 / 73,6.8 \%)$; 
in addition, one patient was treated with a tyrosine-kinase inhibitor $(1 / 73,1.4 \%)$. Choice of regimen was at treating physician's discretion. Treatment was administered for a median of 4.6 cycles (range $=1-8$ cycles). Among patients treated with platinum, cisplatin was used in $18(24.6 \%)$ patients and carboplatin was used in $49(67.1 \%)$ patients. Platinum was combined with either pemetrexed (6 patients), a taxane (26 patients), gemcitabine (26 patients), vilnorelbine (7 patients) or etoposide (2 patients). Non-platinum-based regimens consisted of either pemetrexed, taxane or vilnorelbine monotherapy. Eleven patients $(15.1 \%)$ received radiotherapy as part of their treatment.

Second line treatment such as pemetrexed, docetaxel, combination paclitaxel/gemcitabine or erlotinib was administered in 57 patients. Four patients were enrolled in clinical trials.

Epidermal growth factor receptor (EGFR) status was tested in 21 out of 34 adenocarcinoma patients. Of those 18 $(85.7 \%)$ tumors were found to be EGFR-negative. In the remaining patients, EGFR testing was not feasible due to tissue unavailability (diagnosis was based on cytology). ALK testing was not standard of care at our institution at the time. Median overall survival (OS) was 9 months, while median progressive free survival (PFS) was 5 months. Table I summarizes patient demographics, disease characteristics and patient survival.

In univariate analysis, responders were found to have higher median OS (10 vs. 8 months respectively, $p=0.06)$ and higher median PFS (7 vs. 3 months respectively, $p<0.001$ ).

Protein expression values. In the total population, the median values of IGF-1 and IGF-2 before treatment were $113.4 \mathrm{ng} / \mathrm{ml}$ and $614.37 \mathrm{ng} / \mathrm{ml}$ respectively. The median pretreatment value of IGF1-BP3 was $11511.95 \mathrm{ng} / \mathrm{ml}$. The median value of IGF1/IGF-BP3 ratio at baseline was 0.01252 .

In the total population, the median values of IGF-1, IGF-2 and IGF1-BP3 after treatment were $125.82 \mathrm{ng} / \mathrm{ml}$, $629.87 \mathrm{ng} / \mathrm{ml}$ and $12355.2 \mathrm{ng} / \mathrm{ml}$, respectively. The median value of IGF-1/IGF-BP3 ratio was 0.01006. Data regarding the values of biomarkers before and after treatment are summarized in Table II. In univariate analysis, the median value of IGF-1 after treatment was higher in responders $v s$. non-responders (130.80 vs. $98.00 \mathrm{ng} / \mathrm{ml}$, respectively $p=0.087$ ), as well the median ratio of IGF-1/IGFBP3 (0.01044 vs. 0.00678 respectively, $p=0.056)$ albeit not of statistical significance.

Further analysis of the change of each biomarker value before and after treatment showed statistically significant differences in IGF-1 expression and IGF-1/IGF-BP3 ratio. The median value of IGF-1 was found to be lower after treatment in the total population (125.82 from $133.4 \mathrm{ng} / \mathrm{ml}, p=0.087$ ), albeit no difference was found between subgroups. The median value of IGF-1/IGF-BP3 ratio was found to be lower after treatment in the total population $(0.01006$ from 0.01252 , $p=0.011$ ). Importantly, the post-treatment value of the ratio was significantly lower in responders $(0.01044$ from 0.01255 , $p=0.02$ ). Although there was also a substantial decrease in the post-treatment median ratio of IGF-1/IGF-BP3 in nonresponders $(0.00678$ vs. 0.01225$)$, this was not statistically significant most probably due to the small number of patients. In Table III, we summarize the $\mathrm{p}$ values with regards to the variation in protein expression values before and after treatment. Changes in $\mathrm{p}$ values of each biomarker before and after treatment are summarized in Table III.

Correlation with patient outcomes. No correlation was found between changes in biomarker values, patient OS and PFS. A trend for statistical significance was observed between IGF-1 levels and OS in responders $(p=0.027)$.

\section{Discussion}

In the present study, we sought to determine the prognostic significance and association with clinical outcome of IGF-1, IGF-2 and IGF-BP3 protein expression levels in blood samples from patients with stage IIIB-IV NSCLC treated with 1st line chemotherapy or radical chemoradiation (for patients with stage IIIB disease). A statistically significant reduction in IGF-I protein expression levels, as well as in IGF-I/IGF$\mathrm{BP} 3$ ratio after treatment was found. Most importantly, the decrease in IGF-1/IGF-BP3 ratio, which is a surrogate marker for IGF pathway activation, was found to be statistically lower, only among responders. Our findings support a role of these biomarkers in the evaluation of response to first-line chemotherapy/chemoradiotherapy in patients with recurrent/metastatic and locally advanced NSCLC.

As a central regulator of growth and survival, deregulation of the IGF system is common in human cancer, including NSCLC. IGF1 is the main mediator of the effects of growth hormone $(\mathrm{GH})$; therefore, it has a strong impact on cell proliferation. In a case-control analysis that included 204 lung cancer patients and 218 matched control subjects, Yu et al. have found that increased plasma levels of IGF1 were associated with increased risk for lung cancer $[\mathrm{OR}=2.06$; $95 \%$ confidence interval $(\mathrm{CI})=1.19-3.56 ; p=0.01]$. On the contrary, high plasma levels of IGFBP-3 were associated with reduced risk for lung cancer $(\mathrm{OR}=0.48$; 95\% $\mathrm{CI}=0.25$ $0.92 ; p=0.03)$, and IGF-2 was not associated with lung cancer risk (16). The same group has subsequently published a lung cancer case-control study nested in the placebo arm of the beta-Carotene and Retinol Efficacy Trial in heavy smokers, which did not support the conclusions of the previous report (17). Importantly, two meta-analyses of nested case-control studies and other case-control studies failed to confirm a significant association of IGF-1 and lung cancer but demonstrated an inverse correlation between IGBP3 and lung cancer incidence $(6,18)$. 
Although several studies have assessed the association of circulating concentrations of several components of the IGF axis with lung carcinoma risk over the last years, little is known regarding the predictive role of the IGF-mediated pathway activation in patients with lung cancer. In the present study, we show a significant reduction in IGF-I protein expression levels post-treatment. Although there was no significant difference between responders to treatment and non-responders, this may be attributed to the small sample size. Consequently, IGF-1 alone cannot be suggested as a predictive biomarker for response to treatment in NSCLC. On the other hand, a significant reduction in the IGF-1/IGFP3 ratio was observed in patients who responded to therapy, as compared to non-responders. IGFBP3 is the most abundant carrier protein of IGF-1 in the blood; therefore, the IGF-1/IGFBP3 ratio could be used as an indicator of IGF-1 bioavailability and might be an emerging surrogate predictive marker for chemotherapy efficacy in NSCLC (7). In the era of immunotherapy, there is an unmet need for the discovery of predictive biomarkers of the response to chemotherapy to guide appropriate treatment selection. IGF/IGBP3 ratio could serve as a useful predictive tool in everyday clinical practice for patient selection, as it could be easily measured in peripheral blood at different time points.

In vitro studies conducted in cancer cell lines have demonstrated a correlation between increased IGF-1 levels and resistance to oxaliplatin (19-20). However, few in vivo studies have focused on the predictive and prognostic role of IGF-1 in NSCLC with conflicting results. In a study by Han et al., pretreatment levels of IGF-1 and IGFBP-3 were shown to be favorable prognostic markers in patients with advanced NSCLC treated with cisplatin and irinotecan (21). In another study, increased IGF-1 pretreatment levels were associated with poor response to gefitinib irrespectively of EGFR mutational status in patients with advanced non-squamous NSCLC (22). Indeed, it has been proposed that IGF signaling is upregulated as a means of compensating for the EGFR blockade, in part contributing to resistance to EGFR tyrosinekinase inhibitors. Similarly, reduced IGFBP3 expression has been implicated in EGFR-TKI resistance (23). Of note, our study included only one patient with EGFR mutation. On the other hand, reduction of IGFBP3 expression by promoter methylation is involved in cisplatin resistance in NSCLC. In cells treated with cisplatin, promoter methylation decreases expression of IGFBP-3, thereby inducing activation of the IGF-1R/PI3K/AKT pathway and resistance (24). Treating H640 NSCLC cells with recombinant human IGFBP-3 can restore sensitivity to cisplatin.

Our study has several strengths. There were strong laboratory and clinical data supporting our interest in exploring components of the IGF axis as biomarkers in NSCLC. The patient cohort was prospectively evaluated and all important patient and tumor characteristics were available and evaluable. A major limitation of our study is the small sample size that limits clinical implementation of our results.

In conclusion, the predictive and prognostic significance of IGF-1, IGF-2 and IGFBP3, components of the IGF pathway, in patients with advanced NSCLC treated with chemotherapy or chemoradiotherapy was evaluated. A significant decrease of IGF-1 and IGF-1/IGFBP3 ratio posttreatment was found. Most importantly, the reduction of IGF1/IGFBP3 ratio was statistically significant only among patients who responded to treatment. If validated in larger cohorts, our results support the use of IGF-1/IGFBP 3 as a predictive tool for response to chemotherapy in NSCLC.

\section{Conflicts of Interest}

The Authors declare no conflict of interest regarding this study.

\section{Authors' Contributions}

IK collected the data and wrote the manuscript. PE co-wrote the manuscript. AP supervised the project. EM: carried out the experiments. DP, HG, GD and SG supervised the project, NK and GM conceived the original idea and designed the study. All Authors read and approved the final manuscript.

\section{Acknowledgements}

The Authors wish to thank all patients and their families for agreeing to participate in the study. The Authors would also like to thank Dr. Dimitris Matthaiou for data statistical analysis.

\section{References}

1 Dela Cruz CS, Tanoue LT and Matthay RA: Lung cancer: Epidemiology, etiology, and prevention. Clin Chest Med 32(4): 605-644, 2011. PMID: 22054876. DOI: 10.1016/j.ccm. 2011.09. 001

2 Siegel RL, Miller KD and Jemal A: Cancer statistics, 2017. CA Cancer J Clin 67(1): 7-30, 2017. PMID: 28055103. DOI: $10.3322 /$ caac. 21387

3 Weroha SJ and Haluska P: The insulin-like growth factor system in cancer. Endocrinol Metab Clin North Am 41(2): 335-350, vi, 2012. PMID: 22682634. DOI: 10.1016/j.ecl.2012.04.014

4 Samani AA, Yakar S, LeRoith D and Brodt P: The role of the igf system in cancer growth and metastasis: Overview and recent insights. Endocr Rev 28(1): 20-47, 2007. PMID: 16931767. DOI: $10.1210 / \mathrm{er} .2006-0001$

5 Simpson A, Petnga W, Macaulay VM, Weyer-Czernilofsky U and Bogenrieder T: Insulin-like growth factor (IGF) pathway targeting in cancer: Role of the igf axis and opportunities for future combination studies. Target Oncol 12(5): 571-597, 2017. PMID: 28815409. DOI: 10.1007/s11523-017-0514-5

6 Chen B, Liu S, Xu W, Wang X, Zhao W and Wu J: IGF-I and IGFBP-3 and the risk of lung cancer: A meta-analysis based on nested case-control studies. J Exp Clin Cancer Res 28: 89, 2009. PMID: 19549343. DOI: 10.1186/1756-9966-28-89 
7 Baxter RC: IGF binding proteins in cancer: Mechanistic and clinical insights. Nat Rev Cancer 14(5): 329-341, 2014. PMID: 24722429. DOI: $10.1038 / \mathrm{nrc} 3720$

8 Tas F, Bilgin E, Tastekin D, Erturk K and Duranyildiz D: Serum IGF-1 and IGFBP-3 levels as clinical markers for patients with lung cancer. Biomed Rep 4(5): 609-614, 2016. PMID: 27123 256. DOI: $10.3892 / \mathrm{br} .2016 .629$

9 Denduluri SK, Idowu O, Wang Z, Liao Z, Yan Z, Mohammed MK, Ye J, Wei Q, Wang J, Zhao L and Luu HH: Insulin-like growth factor (IGF) signaling in tumorigenesis and the development of cancer drug resistance. Genes Dis 2(1): 13-25, 2015. PMID: 25984556. DOI: 10.1016/j.gendis.2014.10.004

10 Sun Y, Zheng S, Torossian A, Speirs CK, Schleicher S, Giacalone NJ, Carbone DP, Zhao Z and Lu B: Role of insulinlike growth factor-1 signaling pathway in cisplatin-resistant lung cancer cells. Int J Radiat Oncol Biol Phys 82(3): e563-572, 2012. PMID: 22197230. DOI: 10.1016/j.ijrobp.2011.06.1999

11 Park JH, Choi YJ, Kim SY, Lee JE, Sung KJ, Park S, Kim WS, Song JS, Choi CM, Sung YH, Rho JK and Lee JC: Activation of the igf1r pathway potentially mediates acquired resistance to mutant-selective 3rd-generation egf receptor tyrosine kinase inhibitors in advanced non-small cell lung cancer. Oncotarget 7(16): 22005-22015, 2016. PMID: 26980747. DOI: 10.18632/ oncotarget.8013

$12 \mathrm{Hu}$ Q, Zhou Y, Ying K and Ruan W: IGFBP, a novel target of lung cancer? Clin Chim Acta 466: 172-177, 2017. PMID: 28104361. DOI: 10.1016/j.cca.2017.01.017

13 King H, Aleksic T, Haluska P and Macaulay VM: Can we unlock the potential of igf- $1 \mathrm{r}$ inhibition in cancer therapy? Cancer Treat Rev 40(9): 1096-1105, 2014. PMID: 25123819. DOI: 10.1016/ j.ctrv.2014.07.004

14 Goldstraw P, Crowley J, Chansky K, Giroux DJ, Groome PA, Rami-Porta R, Postmus PE, Rusch V and Sobin L; International Association for the Study of Lung Cancer International Staging Committee; Participating Institutions: The IASLC Lung Cancer Staging Project: Proposals for the revision of the TNM stage groupings in the forthcoming (seventh) edition of the tnm classification of malignant tumours. J Thorac Oncol 2(8): 706-714, 2007. PMID: 17762336. DOI: 10.1097/JTO.0b013e31812f 3c1a

15 Therasse P, Arbuck SG, Eisenhauer EA, Wanders J, Kaplan RS, Rubinstein L, Verweij J, Van Glabbeke M, van Oosterom AT, Christian MC and Gwyther SG: New guidelines to evaluate the response to treatment in solid tumors. European organization for research and treatment of cancer, national cancer institute of the united states, national cancer institute of canada. J Natl Cancer Inst 92(3): 205-216, 2000. PMID: 10655437. DOI: 10.1093/ jnci/92.3.205

16 Yu H, Spitz MR, Mistry J, Gu J, Hong WK and Wu X: Plasma levels of insulin-like growth factor-i and lung cancer risk: A case-control analysis. J Natl Cancer Inst 91(2): 151-156, 1999. PMID: 9923856. DOI: 10.1093/jnci/91.2.151

17 Spitz MR, Barnett MJ, Goodman GE, Thornquist MD, Wu X and Pollak M: Serum insulin-like growth factor (IGF) and IGFbinding protein levels and risk of lung cancer: A case-control study nested in the beta-carotene and retinol efficacy trial cohort. Cancer Epidemiol Biomarkers Prev 11(11): 1413-1418, 2002. PMID: 12433720.
18 Cao H, Wang G, Meng L, Shen H, Feng Z, Liu Q and Du J: Association between circulating levels of igf- 1 and igfbp-3 and lung cancer risk: A meta-analysis. PLoS One 7(11): e49884, 2012. PMID: 23185474. DOI: 10.1371/journal.pone.0049884

19 Volkova E, Robinson BA, Willis J, Currie MJ and Dachs GU: Marginal effects of glucose, insulin and insulin-like growth factor on chemotherapy response in endothelial and colorectal cancer cells. Oncol Lett 7(2): 311-320, 2014. PMID: 24396438. DOI: $10.3892 / \mathrm{ol} .2013 .1710$

$20 \mathrm{Bu}$ Y, Jia QA, Ren ZG, Zhang JB, Jiang XM, Liang L, Xue TC, Zhang QB, Wang YH, Zhang L, Xie XY and Tang ZY: Maintenance of stemness in oxaliplatin-resistant hepatocellular carcinoma is associated with increased autocrine of igf1. PLoS One 9(3): e89686, 2014. PMID: 24632571. DOI: 10.1371/ journal.pone.0089686

21 Han JY, Choi BG, Choi JY, Lee SY and Ju SY: The prognostic significance of pretreatment plasma levels of insulin-like growth factor (IGF)-1, IGF-2, and IGF binding protein-3 in patients with advanced non-small cell lung cancer. Lung Cancer 54(2): 227234, 2006. PMID: 16935391. DOI: 10.1016/j.lungcan. 2006.07.014

22 Masago K, Fujita S, Togashi Y, Kim YH, Hatachi Y, Fukuhara A, Nagai H, Irisa K, Sakamori $Y$, Mio $T$ and Mishima $M$ : Clinical significance of epidermal growth factor receptor mutations and insulin-like growth factor 1 and its binding protein 3 in advanced non-squamous non-small cell lung cancer. Oncol Rep 26(4): 795-803, 2011. PMID: 21805046. DOI: 10.3892/or.2011.1354

23 Guix M, Faber AC, Wang SE, Olivares MG, Song Y, Qu S, Rinehart C, Seidel B, Yee D, Arteaga CL and Engelman JA: Acquired resistance to egfr tyrosine kinase inhibitors in cancer cells is mediated by loss of igf-binding proteins. J Clin Invest 118(7): 2609-2619, 2008. PMID: 18568074. DOI: 10.1172/ JCI34588

24 Cortes-Sempere M, de Miguel MP, Pernia O, Rodriguez C, de Castro Carpeno J, Nistal M, Conde E, Lopez-Rios F, BeldaIniesta $\mathrm{C}$, Perona $\mathrm{R}$ and Ibanez de Caceres I: IGFBP-3 methylation-derived deficiency mediates the resistance to cisplatin through the activation of the IGFIR/Akt pathway in non-small cell lung cancer. Oncogene 32(10): 1274-1283, 2013. PMID: 22543588. DOI: 10.1038/onc.2012.146 